\title{
COMPARISON OF EXCITON PROPERTIES IN QUANTUM WELL STRUCTURES OF ZnCdSe/ZnSe AND ZnSe/ZnMgSSe
}

\author{
M. Godlewsiki ${ }^{a}$, V.Yu. Ivanov ${ }^{a}$, J.P. Bergman ${ }^{b}$, B. MonemaR ${ }^{b}$, \\ K. LeONARDi ${ }^{c}$, M. Behringer ${ }^{c}$ AND D. HommeL ${ }^{c}$ \\ ${ }^{a}$ Institute of Physics, Polish Academy of Sciences \\ Al. Lotników 32/46, 02-668 Warsaw, Poland \\ ${ }^{b}$ Dept. Phys. \& Meas. Technol., Linköping Univ., 58183 Linköping, Sweden \\ ${ }^{c}$ Institute of Solid State Physics, Bremen University, 28334 Bremen, Germany
}

Properties of excitons in quantum well structures of $\mathrm{ZnCdSe} / \mathrm{ZnSe}$ and $\mathrm{ZnSe} / \mathrm{ZnMgSSe}$ are compared. In ternary ZnCdSe quantum wells and at low temperature excitons are strongly localised. Weaker localization is observed in quantum well structures of $\mathrm{ZnSe} / \mathrm{ZnMgSSe}$. Present studies suggest formation of negatively charged excitons in the latter structures.

PACS numbers: 71.35.-y, 73.20.Mf, 73.20.Dx

In this work we compare photoluminescence (PL) emissions observed at low temperatures in two types of ZnSe-based quantum well (QW) structures grown by molecular beam epitaxy on GaAs substrates. Structures with single ZnSe QW and ZnMgSSe barriers, or with multiple ternary $\mathrm{ZnCdSe} Q W$ s and $\mathrm{ZnSe}$ barriers are investigated. Such heterostructures are at present intensely studied due to their possible application in green-blue color light emitting opto-electronics devices [1].

In Figs. 1 and 2 we compare PL spectra observed for two types of the samples studied. PL from the multiple $(2.2 \mathrm{~nm}, 4.3 \mathrm{~nm}, 7.6 \mathrm{~nm}$ and $11.4 \mathrm{~nm}$ wide) QW structure of $\mathrm{ZnCdSe} / \mathrm{ZnSe}$ shows two clearly resolved components from the $2.2 \mathrm{~nm}$ QW, separated by about $7 \mathrm{meV}$. A two-band character of the PL from wider QWs is also resolved at either higher temperatures (see Fig. 1) or at increased excitation intensity. Also the PL from single $\mathrm{ZnSe} \mathrm{QW}$ has the two-band nature. The high-energy components of the PL in two types of the QW structures have their analogs in absorption/reflection and PL excitation (PLE) spectra and thus can be positively identified as free exciton (FE) transitions. The origin of the low-energy component of the PL is different in two types of the samples studied. In $\mathrm{ZnCdSe} / \mathrm{ZnSe}$ this $\mathrm{PL}$ is reduced in the intensity at increased temperatures, or at increased excitation density, and disappears for temperatures larger than

*fax: 48-22-8430926, e-mail:godlew@ifpan.edu.pl 


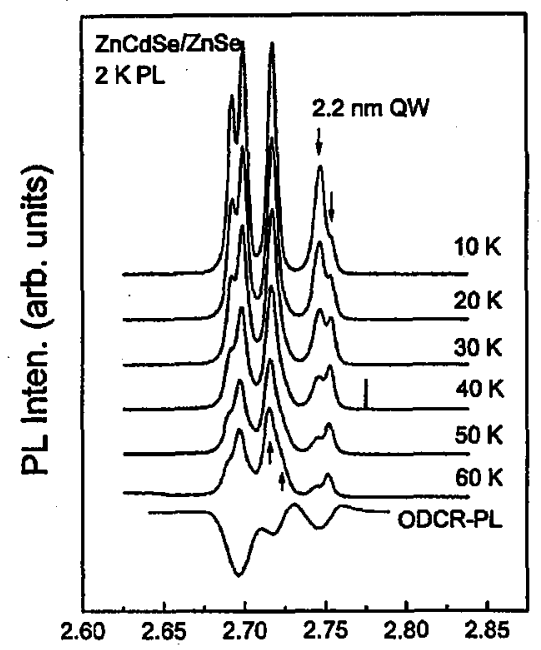

Fig.1 Photon Energy (eV)

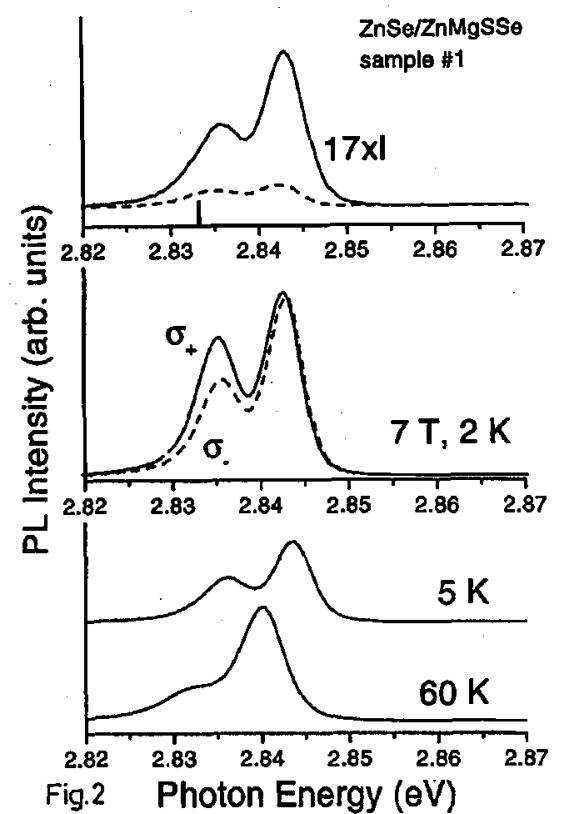

Fig.2 Photon Energy (eV)

Fig. 1. Temperature dependence of the PL spectrum of multiple quantum well structure of $\mathrm{ZnCdSe} / \mathrm{ZnSe}$ consisting of $2.2 \mathrm{~nm}, 4.3 \mathrm{~nm}, 7.6 \mathrm{~nm}$ and $11.4 \mathrm{~nm}$ wide QWs. The two-band structure of the PL from the two narrowest $\mathrm{QWs}$ is indicated with the arrows. The PL response to the microwave power applied at cyclotron resonance conditions (ODCR-PL spectrum) is also shown.

Fig. 2. Photoluminescence spectra of $5 \mathrm{~nm}$ wide $\mathrm{ZnSe} \mathrm{QW}$ in a single $\mathrm{QW} \mathrm{ZnSe} /$ ZnMgSSe structure measured at two temperatures, for two circular polarizations and at two excitation intensities.

about $70 \mathrm{~K}$. Also an interaction with microwave heated free carriers quenches this component of the PL. The high-energy PL is enhanced by an applied microwave power, which results in a derivative-like response of the PL to the microwave radiation (see Fig. 1). Formally, a similar two-band PL emission is observed in the $\mathrm{ZnSe} / \mathrm{ZnMgSSe} \mathrm{QW}$ system (Fig. 2). However, the PL at the lower energy (energy separation of about $8 \mathrm{meV}$ for $5 \mathrm{~nm} \mathrm{QW}$ ) does not saturate with increasing excitation intensity and is more stable at increased temperatures. This PL must thus be of a different origin than that in $\mathrm{ZnCdSe} / \mathrm{ZnSe}$ structures.

There are several possibilities to explain the two-band nature of the PL. Due to an inadvertent contamination with donor species the low-energy component of the PL can be due to the recombination of neutral donor bound excitons (DBEs). If the contamination level is low enough the DBE PL is weak and saturates at increased excitation density. Moreover, DBE PL is thermally deactivated at increased temperatures or by scattering of hot carriers heated by microwave radiation. These properties of DBE emission are often used for its identification. The low-energy component of the PL in the $\mathrm{ZnCdSe}$ QW structures shows such 
properties and is thus attributed to the DBE PL. Quite different properties shows the low-energy component of the two-band PL in the ZnSe/ZnMgSSe QW system. This PL is more stable and is observed up to $100 \mathrm{~K}$. Moreover, its intensity increases with increasing excitation density and the low-energy component of the $\mathrm{PL}$ is not quenched by an applied microwave power. These properties of the PL exclude the DBE origin. We can also exclude that the two-band PL in ZnSe QWs is due to the simultaneous observation of: (a) the $\mathrm{FE}$ and biexciton emissions, (b) the emission from free and localised excitons or (c) the emission of light- and heavy-hole excitons. None of these models is consistent with the present data. For eximple, the biexcitonic emission should increase fast with increasing excitation density. The $8 \mathrm{meV}$ separation of the two PL emissions is inconsistent with the Stokes shift derived from the PLE, which show rather small localization energies in our structures. Thus, the model (b) is incorrect. Moreover, in this model excitons should fast delocalise at increased temperatures and two emissions should merge, which is not observed. The observed energy splitting (and temperature dependence of the PL) also excludes the model (c).

We attribute the low-energy component of the PL in $\mathrm{ZnSe} \mathrm{QWs}$ to the radiative recombination of negatively charged excitons (trions). This, if confirmed, is the first observation of trion PL in the $\mathrm{ZnSe} / \mathrm{ZnMgSSe} \mathrm{QW}$ system. The negatively charged trions consist of two electrons and one hole [2, 3]. Trions were first observed in modulation doped QW structures of CdTe/CdZnTe [3]. There, their origin was concluded from a strong circular polarization of absorption (PL excitation) spectra. However, in our undoped QW structures we do not observe any features in absorption/reflection spectra, which can be related to the trions. Instead, we have studied polarization patterns of the PL using unpolarised or polarised photo-excitation. The strong circular polarization of the low-energy component of the PL is observed with $\sigma^{+}$component of the PL being stronger. A detailed analysis of the PL polarization will be given elsewhere. Here we only conclude that the observed strong circular polarization of the PL (see Fig. 2) and its character are consistent with the trion origin of the low-energy component of the PL. However, lack of supporting absorption/reflection data makes our identification still tentative.

The negatively charged excitons are observed in the present case even though our $\mathrm{ZnSe} / \mathrm{ZnMgSSe}$ structures were not intentionally doped. Their contamination level is low, thus the formation of trions must relate to some differences in properties of the two QW systems studied. The present study indicates relatively weak localization effects in binary ZnSe QWs. A stronger localization of excitons was observed in ternary ZnCdSe QWs [4]. We thus propose that the localization of excitons and carriers may prohibit the formation of trions via trapping of electrons by free excitons. If so, a better morphology of interfaces in $\mathrm{ZnSe} Q W$ structures is here beneficial. The two types of $Q W$ structures also have quite different confinement energies of electrons and holes. The role of increased confinement energies in $\mathrm{ZnSe} / \mathrm{ZnMgSSe}$ structures on the formation rate of trions is however not clear at present.

In magneto-optical studies PL of FEs, DBEs and of trions proceeds ar ong states of different magnetic momenta (spins). In all these cases the PL should be 
circularly polarised. However, a strong PL polarization is observed only for the low-energy component of the PL in the $\mathrm{ZnSe} \mathrm{QWs}$, attributed by us to radiative recombination of trions. From the performed magneto-optical measurements we conclude that the observed strong polarization of the trion PL is due to the spin selection rules for the trions formation and not due to the thermalization among excited states of the trion.

This work was partly supported by grant number 2 P03B 01813 of the Committee for Scientific Research (Poland).

\section{References}

[1] S. Taniguchi, T. Hino, S. Itoh, K. Nakano, N. Nakayama, A. Ishibashi, M. Ikeda, Electron. Lett. 32, 552 (1996).

[2] M.A. Lampert, Phys. Rev. Lett. 1, 450 (1958).

[3] K. Kheng, R.T. Cox, Y. Merle d'Aubigné, F. Basani, K. Saminadayar, S. Tatarenko, Phys. Rev. Lett. 71, 1752 (1993).

[4] M. Godlewski, J.P. Bergman, B. Monemar, E. Kurtz, D. Hommel, Appl. Phys. Lett. 69, 2843 (1996). 